\title{
Strength deficits of the hamstrings following surgery on the anterior cruciate ligament: a case- control study of elite alpine ski racers
}

\section{ELITE SPORTS / LIGAMENT / MUSCLE / RTS / RTS / SPORTS INJURY / SPORTS PHYSIOTHERAPY}

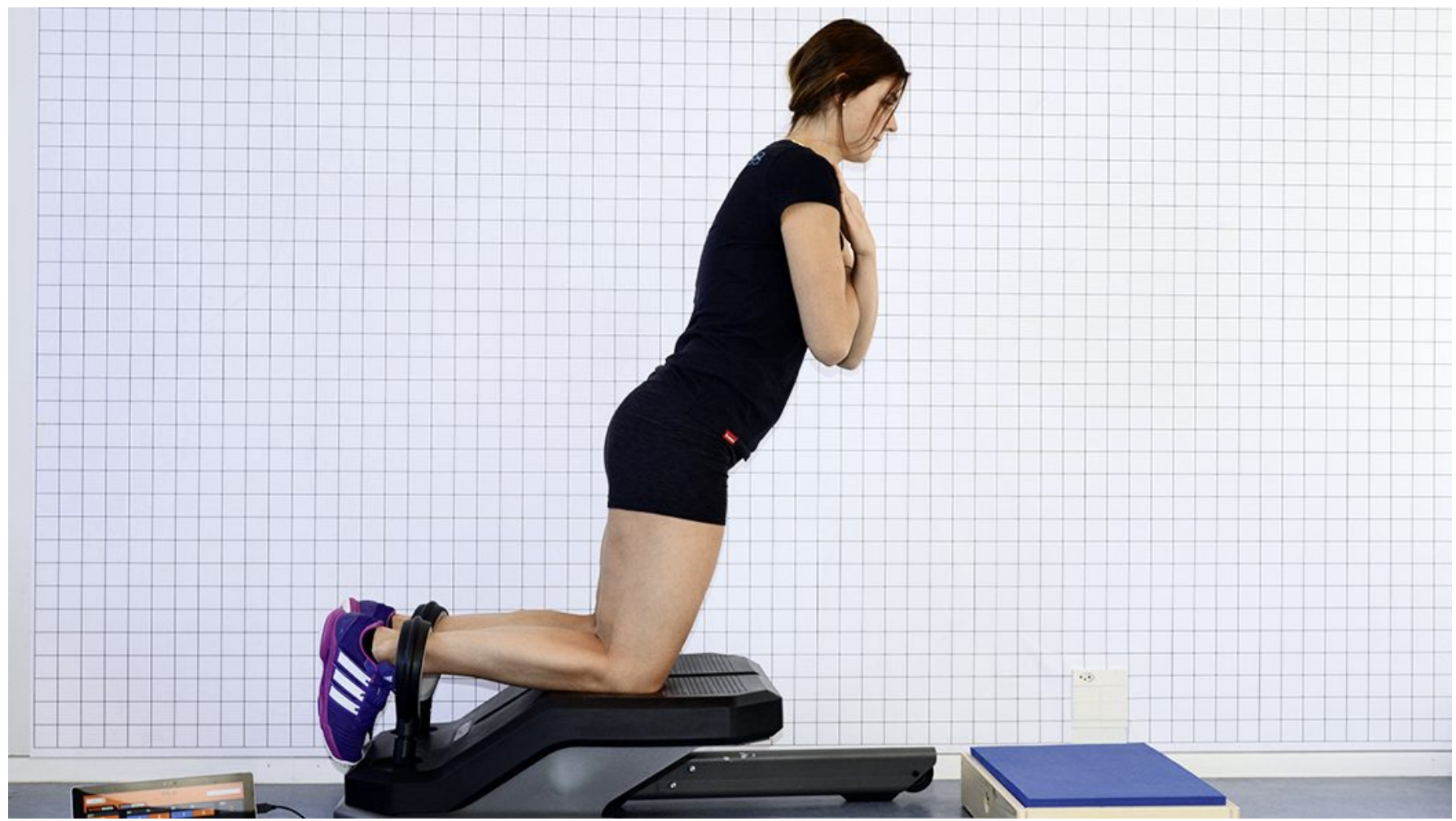

Spiess $\mathrm{J}^{1}$, Meyer $\mathrm{S}^{1}$, Wyss $\mathrm{T}^{1}$, Hübner $\mathrm{K}^{1}$, Bruhin $\mathbf{B}^{1,2}$, Luomajoki $\mathrm{H}^{3}$

${ }^{1}$ Swiss Olympic Medical Center, Eidgenössische Hochschule für Sport Magglingen (EHSM), Bundesamt für Sport (BASPO), Magglingen, Schweiz

${ }^{2}$ Schweizerischer Skiverband Swiss Ski, Muri bei Bern, Schweiz

${ }^{3}$ Zürcher Hochschule für Angewandte Wissenschaften ZHAW, Departement Gesundheit, Institut für Physiotherapie, Winterthur, Schweiz

\section{Abstract}

Background: Research to date clearly shows that injuries to the cruciate ligaments are a frequent occurrence in alpine ski racing. The hamstrings play an important role in protecting the anterior cruciate 
ligament (ACL). Here eccentric muscle activity, in particular, is of great significance as it plays a more important role in terms of duration and intensity than concentric muscle activity in alpine ski racing. The aim of this study was to establish whether the hamstrings of alpine ski racers show eccentric strength deficits following surgery on the anterior cruciate ligament.

Methods: A case-control study was performed with athletes who had undergone surgery on the anterior cruciate ligament $49 \pm 41$ months previously and healthy ski racers. The total eccentric strength of both legs measured in the hamstrings supporting the ACL $(n=18)$ was compared with the healthy group ( $n=70)$; the strength of the operated leg was also compared with the healthy leg and with the mean for the healthy group. Eccentric strength was measured during the Nordic hamstrings exercise performed on the NordBord hamstring testing system. Group comparisons were performed using ANOVA and Bonferroni Post-hoc analyses. Multiple linear regressions were used to assess the relevant independent variables explaining total eccentric strength and Limb Symmetry Index.

Results: The relative total eccentric strength of both legs in the hamstrings of the ACL group was significantly lower than in the healthy group ( $\mathrm{p}=0.031$ ). In addition, the relative eccentric strength of the operated leg was significantly lower compared to the healthy group $(\mathrm{p}=0.024)$. Leg asymmetry was also significantly greater in athletes who had undergone surgery on one leg $(\mathrm{p}=0.016)$ than in healthy subjects and athletes who had undergone surgery on both legs. 56\% of the total eccentric strength of both legs could be explained by surgery, age and gender. $18 \%$ of the leg asymmetry was explained by the variables surgery, total eccentric strength of both legs and height.

Conclusion: Several years after anterior cruciate ligament surgery, ski racers still have deficits in terms of the eccentric strength of their hamstrings compared with healthy ski racers. Therefore more attention should be paid during rehabilitation to the eccentric strength of the hamstrings with a view to strengthening and testing them. Measurement using the NordBord system is suitable for routine monitoring of eccentric strength. Further studies are needed to improve understanding of the influence of hamstrings strength on cruciate ligament injuries.

\section{Zusammenfassung}

Hintergrund: Der Stand der Forschung zeigt auf, dass Kreuzbandverletzungen im alpinen Skirennsport häufig vorkommen. Der ischiocruralen Muskulatur wird eine wichtige Rolle zum Schutz des vorderen Kreuzbandes (VKB) attestiert. Dabei ist v.a. die exzentrische Muskelaktivität von grosser Bedeutung, da sie in Dauer und Intensität gegenüber der konzentrischen Muskelaktivität im alpinen Skirennsport vorherrschend ist. Ziel dieser Studie war herauszufinden, ob Elite-Skirennfahrer nach einer VKB-Plastik exzentrische Kraftdefizite der ischiocruralen Muskulatur aufweisen.

Methode: Es wurde eine Case-Control-Studie zwischen Skirennfahrern $49 \pm 41$ Monaten postoperativ nach einer VKB-Plastik und gesunden Skirennfahrern durchgeführt. Im Seitenvergleich wurde die exzentrische Gesamtkraft beider Beine der ischiocruralen Muskulatur der VKB- $(n=18)$ mit der Kontrollgruppe ( $n=70)$, ferner die Kraft des operierten Beines einerseits mit der gesunden Seite und andererseits mit dem Mittelwert der gesunden Gruppe verglichen. Die exzentrische Kraft wurde während der Übung NordicHamstrings auf dem NordBord gemessen. Gruppenvergleiche wurden mittels ANOVA mit Bonferroni-Posthoc-Tests analysiert. Mittels multipler linearer Regressionsanalyse wurden zudem die relevanten 
unabhängigen Einflussvariablen zur Erklärung der exzentrischen Gesamtkraft und des Limb Symmetry Index ermittelt.

Ergebnisse: Die relative exzentrische Gesamtkraft beider Beine der ischiocruralen Muskulatur der VKBGruppe war signifikant geringer als diejenige der Kontrollgruppe ( $\mathrm{p}=0.031)$. Weiter war das operierte Bein bei der relativen exzentrischen Kraft signifikant schwächer als der Mittelwert der Kontrollgruppe $(\mathrm{p}=0.024)$. Auch der Limb Symmetry Index war bei den unilateral Operierten signifikant grösser $(\mathrm{p}=0.016)$ als bei den Gesunden und den bilateral Operierten. Die Unterschiede in der exzentrischen Gesamtkraft zwischen den Athleten liess sich zu 56\% durch die drei unabhängigen Variablen Operation, Alter und Geschlecht erklären. Die Unterschiede im Limb Symmetry Index zwischen den Athleten konnte zu 18\% durch die drei unabhängigen Variablen Operation, exzentrische Gesamtkraft beider Beine und Körpergrösse erklärt werden.

Schlussfolgerung: Skirennfahrer haben auch mehrere Jahre nach einer VKB-Plastik persistierende Defizite in der exzentrischen Kraft der ischiocruralen Muskulatur verglichen mit gesunden Skirennfahrern. Daher sollte dem Training der exzentrischen Kraftfähigkeit der ischiocruralen Muskulatur während der Rehabilitation aus Sicht der Autoren mehr Aufmerksamkeit gewidmet werden. Die vorgestellte Messmethode ist einfach, praktikabel und kostengünstig. Um den Einfluss der Kraft der ischiocruralen Muskulatur auf Kreuzbandverletzungen besser verstehen zu können, sind weitere Studien nötig.

\section{Einleitung}

Im alpinen Skirennsport sind Knieverletzungen fast ein Drittel der Verletzungen bei Eliteskifahrern [1]. Dabei ist die Verletzung des vorderen Kreuzbandes (VKB) die häufigste Knieverletzung, und Skirennfahrer haben ein hohes Risiko für eine Re-Ruptur [2].

Der ischiocruralen Muskulatur wird eine wichtige Rolle zum Schutz des vorderen Kreuzbandes attestiert [3]. Sie kann der anterioren Translation der Tibia relativ zum Femur entgegenwirken und somit das vordere Kreuzband synergistisch unterstützen [3]. Im Skirennsport könnte dies zur Prävention von VKBVerletzungen speziell relevant sein, da es einen sportartspezifischen Unfallmechanismus mit vergrösserter anteriorer Translationskraft auf die Tibia gibt [1].

Da die exzentrische Muskelaktivität in Dauer und Intensität gegenüber der konzentrischen Muskelaktivität im alpinen Skirennsport vorherrschend ist [4], sollte auch diese Kontraktionsform bei Kraftmessungen erfasst werden. Für die Kraftmessung der ischiocruralen Muskulatur gilt die isokinetische Kraftmessung als Goldstandard [5]. Diese Messmethode ist aber limitiert durch die hohen Kosten und die verminderte Verfügbarkeit des Geräts sowie die zeitaufwendige Durchführung des Tests [6]. Weiter bestehen methodische Probleme wie eingelenkige Messung in offener Kette bei gleichbleibender Geschwindigkeit, was nicht typisch für Bewegungen im Leistungssport ist, die eher mehrgelenkig und beschleunigend bzw. verzögernd sind. Mit einer neuen und einfachen Methode zur Messung der exzentrischen Kraft der hinteren Oberschenkelmuskulatur wurde aufgezeigt, dass hohe bis moderate Level (ICC 0.83-0.9) von TestRetest-Reliabilität erreicht werden können [6]. Bei diesem Testverfahren führt der Proband in einer knienden Position eine Nordic-Hamstrings-Übung auf dem Messgerät aus. Alle Anteile der ischiocruralen Muskulatur sind dabei aktiv, wobei der M. semitendinosus im Vergleich zu den anderen signifikant mehr 
aktiviert wird [7]. Durch die fixe Position des Hüftgelenks weist der M. semitendinosus die günstigsten Hebelverhältnisse auf im Vergleich zum M. bizeps femoris und M. semimembranosus [7]. Dies ist aus zwei Gründen interessant: Einerseits wird der M. semitendinosus bei Skirennfahrern oftmals als Transplantat für das zu ersetzende Kreuzband gewählt und andererseits ist die mediale ette der Hamstrings hauptverantwortlich für das Auffangen von Valgus- und Aussenrotationskräften am Kniegelenk [8]. In der Literatur findet sich nur eine Studie, welche die exzentrische Kraft der ischiocruralen Muskulatur nach einer VKB-Operation bei Athleten mittels NordBord untersucht hat [9]. Es konnte eine signifikant reduzierte exzentrische Kraft der ischiocruralen Muskulatur am operierten Bein im Vergleich zum nicht operierten Bein aufgezeigt werden. Die VKB-Probandengruppe war mit 15 Athleten relativ klein, und die Probanden waren keine Skirennfahrer, sondern Fussball- und Australien Footballspieler. Eine weitere Studie konnte aufzeigen, dass Skirennfahrer nach einer VKB-Rekonstruktion eine verminderte Kraft der Quadriceps- und ischiocruralen Muskulatur aufweisen [10]. Die Kraft wurde isometrisch gemessen. Die Probandenzahl war mit acht VKB-Athleten aber ebenfalls relativ gering. Nach aktuellem Kenntnisstand existieren zum jetzigen Zeitpunkt keine Studien zum Thema der exzentrischen Kraft der ischiocruralen Muskulatur nach einer VKB-Rekonstruktion im alpinen Skirennsport. Weiter wurde die neue Messtechnik mit dem NordBord bisher nie im alpinen Skirennsport angewendet.

Ziel dieser Studie war daher, mit dieser einfachen Messmethode die exzentrische Kraft der ischiocruralen Muskulatur von Skirennfahrern zu untersuchen, um das Niveau der Kraft von am vorderen Kreuzband operierten und gesunden Athleten zu bestimmen.

\section{Methode}

\section{Studiendesign}

Case-Control-Studie zwischen einer Probandengruppe von am vorderen Kreuzband operierten und von gesunden Skirennfahrern.

\section{Probanden und Probandenauswahl}

Für die Studienteilnahme wurden Skirennläufer der Schweizerischen Nationalkader im Alter zwischen 15 und 31 Jahren rekrutiert. Einschlusskriterien für die VKB-Gruppe waren: mind. sechs Monate postoperativ, keine Vorgeschichte von Verletzungen der ischiocruralen Muskulatur in den letzten 12 Monaten, 100\% trainings- und wettkampffähig. Für die Kontrollgruppe galten folgende Kriterien: Keine Vorgeschichte von VKB-Verletzungen und von Verletzungen der ischiocruralen Muskulatur in den letzten 12 Monaten, 100\% trainings- und wettkampffähig. Die Testung musste schmerzfrei durchführbar sein. Aufklärung und Einverständnis sowie positives Ethikvotum lagen vor (KEK-BE-Nr. 2016-00630).

\section{Messinstrumente}

Die exzentrische Kraft wurde mittels der Übung Nordic-Hamstrings in einer knienden Position auf dem NordBord (Vald Performance, Australien) gemessen (Abb. 1). Dabei sind die Füsse fixiert und über die Fixation mit uniaxialen Kraftmesszellen verbunden. Die Datenerhebung erfolgte gleichzeitig auf beiden Seiten in Newton (N). Um die einzelnen Kraftwerte zwischen den Athleten vergleichen zu können, wurden die absoluten Werte zusätzlich in relative Kraftwerte (N/kg) umgerechnet. Folgendes Berechnungsmodell wurde dabei angewendet (A. Shield, persönliches Gespräch, 30. September 2016): (Exzentrische Kraft der 
ischiocruralen Muskulatur in N x Unterschenkellänge in m): (Körpergewicht in kg x Grösse in kniender Position in m). Als Einheit ergibt sich Nm/kgm, gekürzt N/kg. Dadurch werden die einzelnen Hebelverhältnisse und Körperproportionen untereinander vergleichbar. Die Datenerfassung erfolgte durch die SCOREBORD® Software (Vald Performance, Australien).

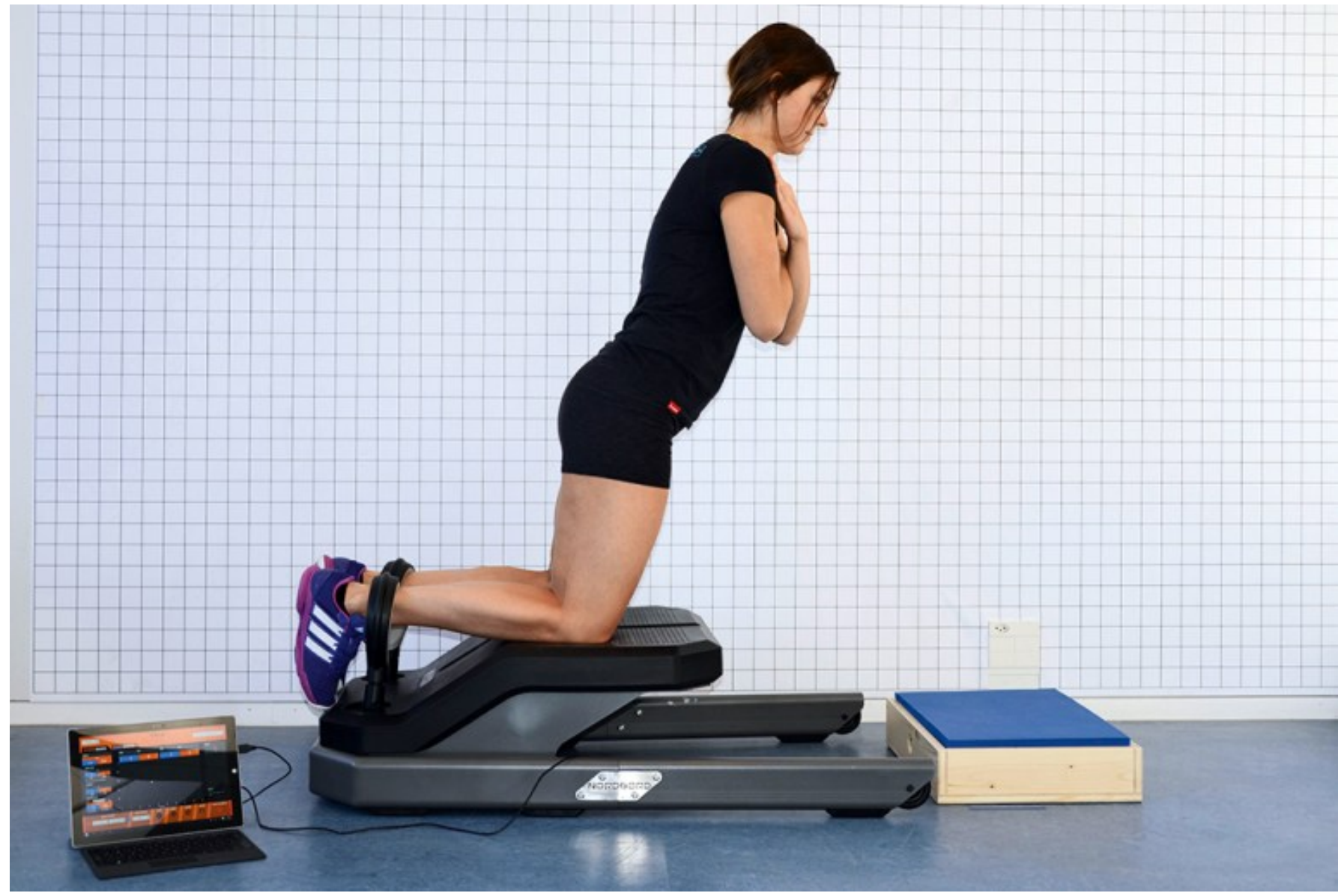

Abb. 1: Testausführung

\section{Testablauf}

Nach Erfassung des Gesundheitszustandes wurde zu Beginn der Testung das Alter, das Körpergewicht (Seca Waage, Modell 877, Hamburg, Deutschland), die Körpergrösse stehend und kniend (Seca Stadiometer, Modell 213, Hamburg, Deutschland) und die Unterschenkellänge nach in der Literatur etablierten Verfahren ermittelt [11,12]. Es folgte ein fünfminütiges Aufwärmen auf dem Fahrradergometer mit anschliessenden Lauf-ABC-Übungen (high-knees und butt-kicking) zur spezifischen Aktivierung der ischiocruralen Muskulatur.

Die Kraftmessung wurde wie in der Literatur beschrieben durchgeführt [6]. Nach einem Warm-up Set von submaximalen Nordic-Hamstringsübungen (spezifisches Aufwärmen auf dem Gerät aber noch relativ früh fallen lassen) erfolgte die bilaterale Messung mit zwei Serien à drei maximalen Nordic-

Hamstringsübungen. Der Tester gab verbale Motivation während der Testung. Wenn die neutrale Position von Rumpf und Hüfte nicht gehalten werden konnte oder das nach vorne Kommen mit dem Oberkörper 
nicht bereits zu Beginn mit den Hamstrings kontrolliert wurde, wurde der Test wiederholt. Die Pausendauer zwischen den Serien betrug zwei Minuten. Die Datenerhebung erfolgte stets durch den gleichen Untersucher.

\section{Datenanalyse und Statistik}

Von den jeweils sechs Messungen auf dem NordBord wurde der Mittelwert für die linke und rechte resp. operierte und nicht operierte Seite für die absolute und relative exzentrische Kraft berechnet. Für die Berechnung des Limb Symmetry Index (Bein-Asymmetrie) wurde die Differenz der exzentrischen Kraft zwischen beiden Beinen berechnet (absolut und in Prozent).

Die Überprüfung der Normalverteilung erfolgte mittels des Komolgorow-Smirnov-Tests. Die Kraftmittelwerte wurden mit dem t-Test für zwei unabhängige Stichproben verglichen bzw. wurde eine einfaktorielle Varianzanalyse verwendet. Bei Vergleichen von drei Gruppen erfolgte die Berechnung mittels einfaktoriellem ANOVA gefolgt vom Bonferroni Post-hoc-Test.

Weiter wurde eine multiple lineare Regressionsanalyse durchgeführt, um den Einfluss von mehreren Faktoren einerseits auf die exzentrische Kraft der ischiocruralen Muskulatur und andererseits auf den Limb Symmetry Index zu erfassen. Alle Berechnungen wurden mit dem Statistikprogramm SPSS Version 21.0 (SPSS, Chicago, USA) durchgeführt. Das Signifikanzniveau wurde bei $\mathrm{p} \leq 0.05$ definiert.

\section{Resultate}

\section{Deskriptive Statistik}

70 unverletzte Skirennfahrer aus den verschiedenen Kadern von Swiss Ski Alpin (Schweizerischer Skiverband Swiss Ski) wurden zu Beginn der Saison untersucht.

Aufgrund der sehr spezifischen Gruppenauswahl (Elite-Athleten aus dem alpinen Skirennsport) konnten nur achtzehn Probanden für die VKB-Gruppe rekrutiert werden. Von diesen achtzehn hatten dreizehn das VKB unilateral und fünf bilateral operiert. Bei dreizehn Athleten der VKB-Gruppe wurde als Kreuzbandersatz das Semitendinosus-Transplantat gewählt, bei drei Athleten ein Transplantat der Streckersehnen (einmal Lig. patellae und zweimal Quadricepssehne) und bei zweien wurde das Kreuzband mittels Naht versorgt. Die Knie der bilateral operierten Probanden waren jeweils mit der gleichen Operationsmethode versorgt worden.

Die Begleitverletzungen im Rahmen der primären Verletzung am vorderen Kreuzband deckten sich mit Rapporten von anderen Studien über Skifahrer [13,14] und beinhalteten Meniskusverletzungen, Innenund Aussenbandverletzungen sowie subchondrale Knochenprellungen. Neun Athleten hatten eine oder mehrere Begleitverletzungen und neun eine isolierte VKB-Ruptur. Von den neun Athleten mit Begleitverletzungen hatten vier eine Verletzung des Aussenmeniskus und fünf mehrere Begleitverletzungen. Die anthropometrischen Daten sind der Tabelle 1 zu entnehmen. Der KolmogorowSmirnov-Test zeigte, dass die Daten bei einer Variablen annäherungsweise (exzentrische Gesamtkraft beider Beine $\mathrm{p}=0.025)$ und bei allen anderen Variablen gut normalverteilt waren $(\mathrm{p}>0.05)$. 


\begin{tabular}{|c|c|c|c|c|c|c|}
\hline & \multicolumn{2}{|l|}{ Alle ( $n=88)$} & \multicolumn{2}{|c|}{ VKB $^{1}$ - Gruppe $(n=18)$} & \multicolumn{2}{|c|}{ Kontrollgruppe $(n=70)$} \\
\hline & Mittelwert & $\mathrm{SD}^{2}$ & Mittelwert & $\mathrm{SD}^{2}$ & Mittelwert & $S^{2}$ \\
\hline Alter (Jahre) & 19.72 & 3.35 & 22.11 & 3.97 & 19.10 & 2.90 \\
\hline Körpergrösse $(\mathrm{cm})$ & 173.14 & 0.08 & 172.07 & 0.08 & 173.42 & 0.08 \\
\hline Körpergrösse kniend (cm) & 130.81 & 0.06 & 129.82 & 0.05 & 131.06 & 0.06 \\
\hline Unterschenkellänge $[\mathrm{cm}]$ & 41.90 & 0.02 & 41.49 & 0.03 & 42.01 & 0.02 \\
\hline Gewicht [kg] & 70.52 & 9.11 & 70.51 & 8.07 & 70.52 & 9.41 \\
\hline Geschlecht w/m³ (Anzahl) & $40 / 48$ & & $11 / 7$ & & $29 / 41$ & \\
\hline $\begin{array}{l}\text { VKB-Transplantat [Beuger/ } \\
\text { Strecker/Naht] }\end{array}$ & & & $13 / 3 / 2$ & & & \\
\hline Anzahl OP $[1$ Bein/2 Beine) & & & $13 / 5$ & & & \\
\hline Zeit seit $\mathrm{OP}^{4}$ [in Monaten] & & & 48.52 & 41.3 & & \\
\hline $\begin{array}{l}\text { Begleitverletzungen } \\
\text { [ja/nein] }\end{array}$ & & & $9 / 9$ & & & \\
\hline $\begin{array}{l}1 \text { Vorderes Kreuzband } \\
2 \text { Standardabweichung } \\
3 \text { w=Frauen, m=Männer } \\
4 \text { Operation }\end{array}$ & & & & & & \\
\hline
\end{tabular}

Tab. 1: Studienpopulation

\section{Exzentrische Gesamtkraft beider Beine}

Die absolute exzentrische Gesamtkraft beider Beine (Summe der exzentrischen Kraft beider Beine pro Athlet) in Newton (N) unterschied sich nicht signifikant zwischen der VKB- und der Kontrollgruppe $(\mathrm{p}=0.078$ ). Ein $\mathrm{p}$-Wert von unter 0.1 deutete aber tendenziell an, dass die Probanden der Kontrollgruppe mehr Kraft erzeugten (Tab. 2).

Bei der Berechnung der relativen exzentrischen Kraft in N/kg unterschieden sich die beiden Gruppen dagegen signifikant $(p=0.031)$.

\section{Vergleich operiertes Bein vs. nicht operiertes Bein}

Bei dieser Fragestellung wurden nur die 13 Probanden innerhalb der VKB-Gruppe berücksichtigt, die unilateral operiert waren (Tab. 3).

Innerhalb der VKB-Gruppe war sowohl bei der absoluten wie auch bei der relativen exzentrischen Kraft kein signifikanter Unterschied zwischen operiertem und nicht operiertem Bein festzustellen ( $\mathrm{p}=0.436$ bei absoluter Kraft; $p=0.305$ bei relativer Kraft). Beim Vergleich der 13 operierten Beine mit dem Mittelwert (zwischen linkem und rechtem Bein) der 70 Probanden der Kontrollgruppe zeigte sich, dass die operierten Beine bei der absoluten Kraft keinen signifikanten Unterschied zur Kontrollgruppe aufweisen ( $p=0.116$ ). Jedoch ergab sich ein signifikanter Unterschied bei der relativen Kraft. Das operierte Bein war schwächer als der Mittelwert der Kontrollgruppe ( $\mathrm{p}=0.024)$. Beim Vergleich zwischen dem gesunden Bein der VKBGruppe mit dem Mittelwert der Kontrollgruppe zeigte sich bei der absoluten und relativen Kraft kein signifikanter Unterschied ( $\mathrm{p}=0.645$ bei absoluter Kraft; $\mathrm{p}=0.462$ bei relativer Kraft). 


\section{Limb Symmetry Index}

Der Limb Symmetry Index unterschied sich zwischen der VKB-Gruppe (unilateral und bilateral) und der Kontrollgruppe signifikant ( $\mathrm{p}=0.016$ ). Wurden drei Gruppen gebildet (Tab. 4), zeigte sich ein signifikanter Unterschied bzgl. des Limb Symmetry Index zwischen der Kontrollgruppe und den unilateral Operierten der VKB-Gruppe ( $\mathrm{p}=0.006$ ). Weiter deuten die Daten einen möglichen, aber nicht signifikanten Unterschied im Limb Symmetry Index zwischen unilateral und bilateral Operierten an $(p=0.121)$. Dieser Unterschied ist aufgrund der kleinen Fallzahl an bilateral Operierten Athleten nicht signifikant ausgefallen.

Hingegen zeigten die bilateral Operierten keinen grösseren Limb Symmetry Index als die Kontrollgruppe $(\mathrm{p}=1.000)$.

Die unilateral operierten Athleten hatten einen Limb Symmetry Index resp. eine Bein-Asymmetrie von 10.44\%. Die bilateral Operierten 5.29\% und die Kontrollgruppe 5.36\%. Abb. 2 zeigt die unterschiedliche Verteilung des Limb Symmetry Index bzgl. operiertem und nicht operiertem Bein der 13 Athleten, die nur an einem Bein operiert waren.

\begin{tabular}{|c|c|c|c|c|c|}
\hline & \multicolumn{2}{|c|}{$\begin{array}{l}\text { VKB' - Gruppe } \\
n=18\end{array}$} & \multicolumn{2}{|c|}{$\begin{array}{l}\text { Kontrollgruppe } \\
n=70\end{array}$} & \multirow{2}{*}{$\begin{array}{l}\text { t-Test für unabhängige } \\
\text { Stichproben } \\
\text { p-Wert }\end{array}$} \\
\hline & Mittelwert & $S^{2}$ & Mittelwert & $\mathrm{SD}^{2}$ & \\
\hline Absolute Kraft [N] & 614.11 & 145.92 & 679.11 & 136.08 & 0.078 \\
\hline Relative Kraft (N/kg) & 2.99 & 0.48 & 3.24 & 0.42 & $0.031^{*}$ \\
\hline \multicolumn{6}{|c|}{$\begin{array}{l}1 \text { Vorderes Kreuzband } \\
2 \text { Standardabweichung } \\
\text { * Signifikanter Unterschied }\end{array}$} \\
\hline
\end{tabular}

Tab. 2: Exzentrische Gesamtkraft beider Beine

\begin{tabular}{|c|c|c|c|c|c|}
\hline & \multirow[t]{2}{*}{ Bein } & \multicolumn{2}{|c|}{ Absolute Kraft (N) } & \multicolumn{2}{|c|}{ Relative Kraft (N/kg) } \\
\hline & & Mittelwert & $\mathrm{SD}^{2}$ & Mittelwert & $\mathrm{SD}^{2}$ \\
\hline \multirow[t]{2}{*}{ VKB 1 -Gruppe [n=13] } & Operiertes Bein & 320.60 & 105.99 & $1.45^{*}$ & 0.41 \\
\hline & Gesundes Bein & 347.21 & 58.42 & 1.57 & 0.17 \\
\hline Kontrollgruppe $(n=70]$ & Mittelwert li/re Bein & 356.52 & 67.92 & $1.62^{*}$ & 0.21 \\
\hline
\end{tabular}




\begin{tabular}{|c|c|c|c|c|}
\hline & n & Asymmetrie $M W^{1}$ (N) & $\mathrm{SD}^{2}(\mathrm{~N})$ & Rel. Asymmetrie $[\%]$ \\
\hline Kontrollgruppe & 70 & 36.40 & 26.17 & 5.36 \\
\hline Unilateral Operierte & 13 & 64.04 & 41.63 & 10.44 \\
\hline Bilateral Operierte & 05 & 32.60 & 21.46 & 5.29 \\
\hline Insgesamt & 88 & 40.27 & 30.05 & 6.05 \\
\hline
\end{tabular}

Tab. 4: Zusammenfassung des Limb Symmetry Index (absolut und in Prozent) der einzelnen Gruppen

\section{Einflussfaktoren auf die exzentrische Gesamtkraft beider Beine}

Die beiden zu vergleichenden Stichproben unterscheiden sich bezüglich Faktoren, welche die Beinkraft beeinflussen können, wie Alter, Frauenanteil und Anthropometrie. Um festzustellen, ob ein Unterschied in der exzentrischen Gesamtkraft zwischen operierten und nicht operierten Athleten bestehen bleibt, wenn der Einfluss dieser Variablen auskorrigiert wird, wurde eine multiple lineare Regressionsanalyse durchgeführt. Folgende Einflussvariablen wurden in die multiple lineare Regressionsanalyse miteinbezogen: Operation, Alter, Zeit seit der Operation, Geschlecht, Körpergrösse, Gewicht und kniende Körpergrösse. Da zwischen der Körpergrösse und der Unterschenkellänge eine Multikollinearität vorlag $(\mathrm{r}=0.846)$, wurde stellvertretend für beide Variablen die Körpergrösse im Modell miteinbezogen. Bilateral operierte Probanden wurden aufgrund der kleinen Fallzahl aus dieser Auswertung ausgeschlossen. Athleten, die unilateral operiert waren, hatten weniger exzentrische Gesamtkraft beider Beine, wenn die Daten nach Geschlecht und Alter korrigiert wurden (Älter=stärker; Männer=stärker). Die Variablen Operation, Alter und Geschlecht erklärten zusammen 56\% der exzentrischen Gesamtkraft beider Beine ( $\mathrm{R} 2=0.577$, korrigiertes $\mathrm{R} 2=0.560, \mathrm{~F}=35.85, \mathrm{p}=0.000$ ). Alle anderen Variablen hatten keinen Einfluss auf die exzentrische Gesamtkraft beider Beine.

Den grössten Einfluss (48\%) auf die exzentrische Gesamtkraft beider Beine der Athleten hatte das Geschlecht. Tab. 5 zeigt den Einfluss der drei Variablen.

\section{Einflussfaktoren auf den Limb Symmetry Index}

Es wurden dieselben unabhängigen Variablen wie unter «Einflussfaktoren auf die exzentrische Gesamtkraft beider Beine» und zusätzlich die Variable exzentrische Gesamtkraft beider Beine miteinbezogen. Erneut wurde die Körpergrösse auch stellvertretend für die Unterschenkellänge mit einbezogen, da eine Multikollinearität zwischen beiden Variablen vorlag ( $\mathrm{r}=0.846)$. Der Limb Symmetry Index wurde zu 18\% durch folgende Variablen erklärt: Exzentrische Gesamtkraft beider Beine, Körpergrösse und Operation (R2=0.206, korrigiertes $\mathrm{R} 2=0.176, \mathrm{~F}=6.85, \mathrm{p}=0.000$ ). Mehr Kraft führte zu weniger Asymmetrie, operiert zu mehr Asymmetrie. Ebenfalls hatte eine zunehmende Körpergrösse mehr Asymmetrie zur Folge (Tab. 6).

\section{Diskussion}

Dies ist die erste Studie, welche die exzentrische Kraft der ischiocruralen Muskulatur bei am vorderen Kreuzband operierten und gesunden Skirennfahrern untersuchte. Solche Untersuchungen sind wichtig 
aufgrund der hohen Inzidenz von VKB-Verletzungen und der hohen Re-Rupturgefahr im Skirennsport und sollen helfen, zunehmend zu verstehen, welche Einflussfaktoren nach Verletzungen und Operationen gegenüber der normalen Situation verändert sind [1,2].

Es erstaunt nicht, dass sich nur bei der relativen exzentrischen Gesamtkraft beider Beine ein Unterschied zwischen der VKB- und der Kontrollgruppe zeigte. Die Formel für die relative Kraft berücksichtigt die Grössen Unterschenkellänge, Gewicht und Körpergrösse in kniender Position, und so werden die Probanden untereinander besser vergleichbar und erlauben eine zuverlässigere Aussage. Das Gleiche gilt beim Vergleich zwischen operiertem Bein und dem Mittelwert der Kontrollgruppe. Zum gleichen Ergebnis kam die Studie von Buchheit et al. [15], welche aber nur das Körpergewicht und nicht die einzelnen Hebelverhältnisse berücksichtigte bei Fussballspielern.

Analog zu den Ergebnissen dieser Studie sind die Resultate von Timmins et al. [9], die ebenfalls zeigten, dass das operierte Bein bei Fussball- und Australien Footballspielern schwächer war als das der Kontrollgruppe. Zusätzlich war das operierte Bein auch signifikant schwächer als das gesunde Bein. Dies konnte bei dieser Studie nicht gezeigt werden, einerseits aufgrund der kleinen Stichprobe (n=13) und andererseits aufgrund der hohen Standardabweichung bei den operierten Beinen (SD=105.99). Die hohe Standardabweichung ist vorhanden, weil offensichtlich das Verhalten nach der Operation resp. die Rehabilitation doch sehr individuell zu sein scheint. Bei der genaueren Betrachtung der dreizehn unilateral Operierten zeigte sich, dass fünf Athleten auf dem operierten Bein stärker waren als auf dem gesunden Bein. Bei den fünf, die auf dem operierten Bein stärker waren, hatten nur zwei ein Transplantat der Beugersehnen, was eine mögliche Erklärung sein könnte. Wurde die exzentrische Gesamtkraft beider Beine aber für Geschlecht und Alter korrigiert, zeigte sich auch, dass die Operation zu einer Verminderung der exzentrischen Kraft führte und somit gesagt werden kann, dass das operierte Bein tendenziell schwächer war als das gesunde Bein des gleichen Athleten.

Der Limb Symmetry Index von 10.44\% bei den unilateral Operierten der VKB-Gruppe ist etwas tiefer als die Resultate von anderen Studien. Timmins et al. [9] berichteten über einen Limb Symmetry Index von 13.7\% und Jordan et al. [10]über einen von 14.6\%. Da alle Studien den Limb Symmetry Index auf eine andere Art berechneten und zusätzlich die Kraft bei der Studie von Jordan et al. (10) nicht exzentrisch gemessen wurde, ist ein korrekter Vergleich kaum möglich. Die Literatur beschreibt einen Limb Symmetry Index von unter 10\% als genügend für die Rückkehr zum Sport [16-18]. Mit den 10.44\% waren die unilateral Operierten der VKB-Gruppe knapp darüber, wobei die klinische Relevanz kritisch hinterfragt werden kann. Im Vergleich zur Kontrollgruppe war die Differenz aber um 5\% höher, und interessanterweise zeigten die bilateral Operierten einen sehr ähnlichen Limb Symmetry Index (5.29\%) wie die Gesunden (5.36\%), jedoch auf einem tieferen Kraftniveau. Dieses Resultat der gesunden Skirennfahrer spricht für die Wichtigkeit einer geringen Asymmetrie der exzentrischen Kraft, was sich durch das Anforderungsprofil der repetitiven bidirektionalen Kurven dieser Sportart erklären lässt [10]. Der Skirennsport verlangt beidseitig gleich gut ausgebildete Beinkraft. Auch die multiple Regressionsanalyse bzgl. des Limb Symmetry Index ergab, dass eine unilaterale Operation zu mehr Asymmetrie führt, wobei aber das operierte Bein nicht immer das schwächere Bein ist. Ein möglicher Grund könnte die Wahl des Transplantates sein. Bei den fünf, die auf dem operierten Bein stärker waren, hatten nur zwei ein Transplantat der Beugersehnen.

Anders als erwartet konnte nicht aufgezeigt werden, dass mehrere Begleitverletzungen zu mehr Asymmetrie führen. So wies der Proband mit den meisten Begleitverletzungen einen Limb Symmetry 
Index von 7\% auf, wogegen der Proband mit «nur» einer isolierten VKB-Ruptur eine Asymmetrie von 55\% hatte. Dies lässt vermuten, dass der Bewegungsablauf der Testausführung keinen Einfluss auf den Schweregrad der Verletzung hat. Da die Probandenanzahl aber relativ klein war, lässt sich hier keine abschliessende Aussage formulieren.

Weiter zeigte sich bei einer stärkeren exzentrischen Kraft ein kleinerer Limb Symmetry Index. Männliche Skirennfahrer haben eine höhere exzentrische Kraftfähigkeit der ischiocruralen Muskulatur als die Frauen. Zum gleichen Resultat kam die Studie von Neumayr et al. [19], welche die Kraft der ischiocruralen Muskulatur bei Skirennfahrern isokinetisch testeten (Cybex, konzentrische Muskelaktion, Winkelgeschwindigkeiten von $60 \%$ und $240 \%$ s). Im Widerspruch zu diesen Resultaten steht die Studie von Jordan et al. [10], die die Kraftfähigkeit von Quadriceps und Hamstrings bei kanadischen EliteSkirennfahrern testeten und keinen Unterschied feststellen konnten. Da die Kraft isometrisch (Isokinetik Biodex Dynamometer, Knie in $70^{\circ}$ Flexion) gemessen wurde und nur an 21 Athleten, ist ein Vergleich der beiden Resultate schwierig.

Die Resultate dieser Studie sind ein wichtiger Informationsgewinn und von grosser klinischer Relevanz für die Rehabilitation von Skirennfahrern nach einer VKB-Plastik. Trotz der Rückkehr zum Sport besteht nach wie vor eine verminderte exzentrische Kraft am operierten Bein (Zeit seit Operation im Mittelwert $48.52 \pm$ 41.3 Monate) bei dieser spezifischen Athletengruppe. In der Studie von Ristanis et al. [20] konnte gezeigt werden, dass eine VKB-Rekonstruktion mittels Semitendinosus-Transplantat zu einer erhöhten Verzögerung der Muskelkontraktion der Kniebeugemuskulatur nach Eintreffen des elektrischen Impulses auf den Muskel führt. Dies könnte wiederum zu einer verminderten Kniestabilität führen [20]. Da in der vorliegenden Studie 13 Athleten ein Semitendinosus-Transplantat hatten, könnte dies eine Erklärung für die verminderte exzentrische Kraft der ischiocruralen Muskulatur sein.

Der exzentrischen Kräftigung der ischiocruralen Muskulatur sollte somit in Zukunft während der Rehabilitation mehr Aufmerksamkeit gewidmet werden. Exzentrisches Krafttraining führt zu einer Verlängerung der Faszikel der Muskulatur und zu einer Kraftzunahme des Muskels [21]. Bei Athleten mit einem Semitendinosus-Transplantat sollten demnach spezifische Übungen gewählt werden, um diesen Muskel der ischiocruralen Kette wieder gezielt zu kräftigen. Bei der Nordic-Hamstrings-Übung konnte eine erhöhte Aktivität dieses Muskels aufgezeigt werden [21]; diese Übung sollte daher zwingend in die Rehabilitation integriert und deren Wirkung im Verlauf immer wieder überprüft werden.

Limitierend für diese Studie ist erstens, dass es nicht möglich war, mehr am vorderen Kreuzband operierte Skirennfahrer zu rekrutieren, um einen Effekt zwischen operiertem und gesundem Bein zu zeigen und um einen Unterschied bzgl. Transplantatentnahme zu untersuchen. Zweitens kann ein durch den nicht verblindeten Tester verursachter Bias nicht ausgeschlossen werden.

Das NordBord-Gerät selber ist dadurch limitiert, dass es die Winkelgeschwindigkeit wie auch den Winkel der Kraftspitze nicht messen und abbilden kann. Die Test-Retest-Reliabilität für den Winkel der Kraftspitze für die Hamstrings gemessen bei isokinetischen Tests ist aber tief, und zum jetzigen Zeitpunkt gibt es in der Literatur keinen Konsens über ein geeignetes Vorgehen [22].

Bei der operativen Gruppe ist das Verhältnis zwischen Frauen und Männern deutlich zugunsten der weiblichen Athleten. In der Kontrollgruppe ist es genau umgekehrt. Deshalb wurden zusätzlich mittels multipler linearer Regression der Einfluss der Operation auf die Gesamtkraft unter Einbezug von Alter und Geschlecht untersucht. So konnte festgestellt werden, dass operierte Personen eine geringere Kraft aufweisen, wenn die Daten nach Alter und Geschlecht korrigiert werden. 


\section{Praktische Relevanz}

- Die Verletzung des vorderen Kreuzbandes ist die häufigste Knieverletzung im Skirennsport.

- Der ischiocruralen Muskulatur wird eine wichtige Rolle zum Schutz des vorderen Kreuzbandes attestiert.

- Skirennfahrer haben auch mehrere Jahre nach einer Operation am vorderen Kreuzband noch Defizite in der exzentrischen Kraft der ischiocruralen Muskulatur im operierten Bein.

- Der exzentrischen Kraftfähigkeit der ischiocruralen Muskulatur sollte während der Rehabilitation mehr Aufmerksamkeit gewidmet werden im Sinne von Kräftigung und Überprüfung.

- Die Messung mit dem NordBord ist eine einfache, praktikable und kostengünstige Messmethode.

\section{Danksagung und Interessenkonflikt}

Es besteht kein Interessenkonflikt.

Die Autoren danken den teilnehmenden Schweizer Skirennfahrern aus den verschiedenen Kadern von Swiss Ski Alpin und den Trainern (speziell Raphael Schmidiger) für die Unterstützung bei der Organisation der Testungen.

Aus Gründen der besseren Lesbarkeit wurde in dieser Studie auf die Nennung beider Geschlechter verzichtet. Es sind aber immer beide Geschlechter gemeint, wenn die männliche Form gewählt wurde.

\section{Korrespondenzadresse}

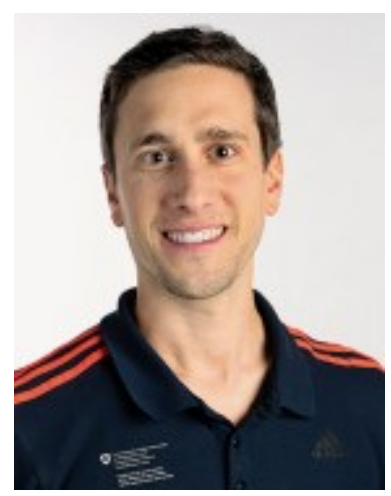

Jonas Spiess PT,

MAS ZFH in Muskuloskelettaler

Physiotherapie

Sportphysiotherapie, Swiss Olympic

Medical Center

Eidgenössisches Departement für

Verteidigung, Bevölkerungsschutz

und Sport VBS $\square$

Bundesamt für Sport BASPO

Eidg. Hochschule für Sport Magglingen EHSM

2532 Magglingen

Tel. +41584676333 
jonas.spiess@baspo.admin.ch

\section{Referenzen}

1. Bere T, Florenes TW, Krosshaug T, Koga H, Nordsletten L, Irving C, et al. Mechanisms of anterior cruciate ligament injury in World Cup alpine skiing: a systematic video analysis of 20 cases. The American journal of sports medicine. 2011;39(7):1421-9.

2. Pujol N, Blanchi MP, Chambat $P$. The incidence of anterior cruciate ligament injuries among competitive Alpine skiers: a 25-year investigation. The American journal of sports medicine. 2007;35(7):1070-4.

3. MacWilliams BA, Wilson DR, DesJardins JD, Romero J, Chao EY. Hamstrings cocontraction reduces internal rotation, anterior translation, and anterior cruciate ligament load in weight-bearing flexion. Journal of orthopaedic research: official publication of the Orthopaedic Research Society. 1999;17(6):817-22.

4. Berg HE, Eiken O. Muscle control in elite alpine skiing. Medicine and science in sports and exercise. 1999;31(7):1065-7.

5. Aagaard P, Simonsen EB, Magnusson SP, Larsson B, Dyhre-Poulsen P. A new concept for isokinetic hamstring: quadriceps muscle strength ratio. The American journal of sports medicine. 1998;26(2):231-7.

6. Opar DA, Piatkowski T, Williams MD, Shield AJ. A novel device using the Nordic hamstring exercise to assess eccentric knee flexor strength: a reliability and retrospective injury study. The Journal of orthopaedic and sports physical therapy. 2013;43(9):636-40.

7. Bourne MN, Opar DA, Williams MD, Al Najjar A, Shield AJ. Muscle activation patterns in the Nordic hamstring exercise: Impact of prior strain injury. Scandinavian journal of medicine \& science in sports. 2016;26(6):666-74.

8. Hewett TE, Myer GD, Ford KR, Heidt RS, Jr., Colosimo AJ, McLean SG, et al. Biomechanical measures of neuromuscular control and valgus loading of the knee predict anterior cruciate ligament injury risk in female athletes: a prospective study. The American journal of sports medicine. 2005;33(4):492-501.

9. Timmins RG, Bourne MN, Shield AJ, Williams MD, Lorenzen C, Opar DA. Biceps Femoris Architecture and Strength in Athletes with a Previous Anterior Cruciate Ligament Reconstruction. Medicine and science in sports and exercise. 2016;48(3):337-45.

10. Jordan MJ, Aagaard P, Herzog W. Rapid hamstrings/quadriceps strength in ACL-reconstructed elite Alpine ski racers. Medicine and science in sports and exercise. 2015;47(1):109-19.

11. Muggleton S. Kinematics and Kinetics of the Nordic Hamstring Curl [Dissertation]. Queensland: School of Exercise and Nutrition Sciences Queensland University of Technology; 2015.

12. Maier T, Gross M, Trösch S, Steiner T, Müller B, Bourban P, et al. Manual Leistungsdiagnostik: Qualitätsentwicklung Sportmed Swiss Olympic Magglingen; 2016.

13. Granan LP, Inacio MC, Maletis GB, Funahashi TT, Engebretsen L. Sport-specific injury pattern recorded during anterior cruciate ligament reconstruction. The American journal of sports medicine. 2013;41(12):2814-8.

14. Paletta GA, Jr., Levine DS, O’Brien SJ, Wickiewicz TL, Warren RF. Patterns of meniscal injury associated with acute anterior cruciate ligament injury in skiers. The American journal of sports medicine. 1992;20(5):542-7.

15. Buchheit M, Cholley Y, Nagel M, Poulos N. The Effect of Body Mass on Eccentric Knee-Flexor Strength Assessed With an Instrumented Nordic Hamstring Device (Nordbord) in Football Players. Int J Sports Physiol Perform. 2016;11(6):721-6.

16. Zwolski C, Schmitt LC, Thomas S, Hewett TE, Paterno MV. The Utility of Limb Symmetry Indices in 
Return-to-Sport Assessment in Patients With Bilateral Anterior Cruciate Ligament Reconstruction. The American journal of sports medicine. 2016;44(8):2030-8.

17. Ardern CL, Webster KE. Knee flexor strength recovery following hamstring tendon harvest for anterior cruciate ligament reconstruction: a systematic review. Orthop Rev (Pavia). 2009;1(2):e12.

18. Schmitt LC, Paterno MV, Ford KR, Myer GD, Hewett TE. Strength Asymmetry and Landing Mechanics at Return to Sport after Anterior Cruciate Ligament Reconstruction. Medicine and science in sports and exercise. 2015;47(7):1426-34.

19. Neumayr G, Hoertnagl H, Pfister R, Koller A, Eibl G, Raas E. Physical and physiological factors associated with success in professional alpine skiing. International journal of sports medicine. 2003; 24(8):571-5.

20. Ristanis S, Tsepis E, Giotis D, Stergiou N, Cerulli G, Georgoulis AD. Electromechanical delay of the knee flexor muscles is impaired after harvesting hamstring tendons for anterior cruciate ligament reconstruction. The American journal of sports medicine. 2009;37(11):2179-86.

21. Bourne MN, Duhig SJ, Timmins RG, Williams MD, Opar DA, Al Najjar A, et al. Impact of the Nordic hamstring and hip extension exercises on hamstring architecture and morphology: implications for injury prevention. Br J Sports Med. 2017;51(5):469-77.

22. Timmins RG, Shield AJ, Williams MD, Opar DA. Is There Evidence to Support the Use of the Angle of Peak Torque as a Marker of Hamstring Injury and Re-Injury Risk? Sports Med. 2016;46(1):7-13. 